\title{
Clinical risk factors of colorectal cancer in patients with serrated polyposis syndrome: a multicentre cohort analysis
}

\author{
J E G IJspeert, ${ }^{1}$ S A Q Rana, ${ }^{2}$ N S S Atkinson, ${ }^{3}$ Y J van Herwaarden, ${ }^{4}$ \\ B A J Bastiaansen, ${ }^{1} \mathrm{M}$ E van Leerdam, ${ }^{5}$ S Sanduleanu, ${ }^{6} \mathrm{~T}$ M Bisseling, ${ }^{4}$ \\ M C W Spaander, ${ }_{1}^{7}$ S K Clark, ${ }^{2}$ G A Meijer, ${ }_{1}^{8}$ N van Lelyveld, ${ }_{1}^{9}$ J J Koornstra, ${ }_{1}^{10}$ \\ I D Nagtegaal, ${ }^{11}$ J E East, ${ }^{3}$ A Latchford, ${ }^{2}$ E Dekker, ${ }^{1}$ on behalf of the Dutch \\ workgroup serrated polyps \& polyposis (WASP)
}

\begin{abstract}
- Additional material is published online only. To view please visit the journal online (http://dx.doi.org/10.1136/ gutjnl-2015-310630).

For numbered affiliations see end of article.
\end{abstract}

\section{Correspondence to} Dr Evelien Dekker, Department of Gastroenterology and Hepatology, Academic Medical Centre, Meibergdreef 9 , Amsterdam $1105 \mathrm{AZ}$, The Netherlands; e.dekker@amc.uva.nl

Received 26 August 2015 Revised 23 September 2015 Accepted 28 October 2015 Published Online First 24 November 2015

\section{CrossMark}

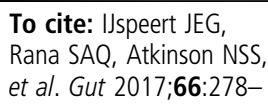

\section{ABSTRACT}

Objective Serrated polyposis syndrome (SPS) is accompanied by an increased risk of colorectal cancer (CRC). Patients fulfilling the clinical criteria, as defined by the WHO, have a wide variation in CRC risk. We aimed to assess risk factors for CRC in a large cohort of patients with SPS and to evaluate the risk of CRC during surveillance.

Design In this retrospective cohort analysis, all patients with SPS from seven centres in the Netherlands and two in the UK were enrolled. WHO criteria were used to diagnose SPS. Patients who only fulfilled WHO criterion2, with IBD and/or a known hereditary CRC syndrome were excluded.

Results In total, 434 patients with SPS were included for analysis; 127 (29.3\%) were diagnosed with CRC. In a per-patient analysis $\geq 1$ serrated polyp (SP) with dysplasia (OR 2.07; 95\% CI 1.28 to 3.33 ), $\geq 1$ advanced adenoma (OR 2.30; $95 \% \mathrm{Cl} 1.47$ to 3.67 ) and the fulfilment of both WHO criteria 1 and 3 (OR $1.60 ; 95 \% \mathrm{Cl} 1.04$ to 2.51 ) were associated with CRC, while a history of smoking was inversely associated with CRC (OR 0.36; 95\% Cl 0.23 to 0.56). Overall, 260 patients underwent surveillance after clearing of all relevant lesions, during which two patients were diagnosed with CRC, corresponding to 1.9 events/1000 person-years surveillance $(95 \% \mathrm{Cl} 0.3$ to 6.4).

Conclusion The presence of SPs containing dysplasia, advanced adenomas and/or combined WHO criteria 1 and 3 phenotype is associated with CRC in patients with SPS. Patients with a history of smoking show a lower risk of CRC, possibly due to a different pathogenesis of disease. The risk of developing CRC during surveillance is lower than previously reported in literature, which may reflect a more mature multicentre cohort with less selection bias.

\section{BACKGROUND}

Colorectal cancer (CRC) is one of the main cancerrelated causes of morbidity and mortality in the Western world. ${ }^{1}$ As CRC arises from premalignant polyps, the detection and resection of these lesions decrease both CRC incidence and mortality. ${ }^{2}$ A growing body of evidence shows that 15\%-30\% of all CRCs arise from serrated polyps (SPs) rather

\section{Significance of this study}

What is already known on this subject?

- Serrated polyposis syndrome is accompanied by an increased risk of colorectal cancer.

- Patients fulfilling the clinical criteria for serrated polyposis syndrome have a wide variation in colorectal cancer risk.

- Colorectal cancer risk factors in these patients are not yet well defined.

\section{What are the new findings?}

- Serrated polyps containing dysplasia, advanced adenomas and/or a combined WHO criteria 1 and 3 phenotype are associated with colorectal cancer in patients with serrated polyposis syndrome.

- Patients with serrated polyposis having a history of smoking show a lower risk of colorectal cancer, possibly due to a different pathogenesis of disease.

- The risk of colorectal cancer during surveillance and after clearing of all relevant lesions is lower than suggested earlier.

How might it impact on clinical practice in the foreseeable future?

- The clinical risk factors that we discovered in the current study could help to risk stratify patients with serrated polyposis for different surveillance intervals in order to decrease patient burden as well as the incidence of colonoscopy interval colorectal cancer. 
and traditional serrated adenomas (TSAs)..$^{10}$ Diminutive HPs located in the rectosigmoid are generally considered benign, whereas larger and/or proximally located HPs, and all SSA/Ps and TSAs are considered to possess a higher neoplastic potential. ${ }^{11} 12$

Serrated polyposis syndrome (SPS) is a syndrome characterised by multiple SPs located throughout the colon and is accompanied by an increased risk of CRC. ${ }^{10}$ The prevalence of SPS in the general population is largely unknown; however, rates in colonoscopy screening populations of 1:2000 have been reported. ${ }^{13}$ In fecal occult blood test (FOBT)-based population screening, the prevalence of SPS may exceed 1:300 participants, demonstrating the importance for endoscopists to recognise and diagnose this syndrome. ${ }^{13}$ As germline mutations for SPS are unknown, this disease has been clinically defined by the WHO by the presence of at least five SPs proximal to the sigmoid colon, of which two are $\geq 10 \mathrm{~mm}$ in diameter (WHO criterion-1), the presence of one SP proximal to the sigmoid and a first-degree relative (FDR) with SPS (WHO criterion-2) and/or 20 SPs or more, irrespective of size, but located throughout the colorectum (WHO criterion-3). ${ }^{10}$ Although WHO criterion-2 is rarely used, this clinical definition of the WHO leads to a very heterogeneous group of patients with SPS, with a wide variation in CRC risk. CRC risk for patients at their first presentation with SPS is reported up to $50 \%$, while several retrospective studies have shown that these patients also have an increased risk of developing CRC under endoscopic surveillance. ${ }^{14-18}$ However, the actual risk of CRC for patients with SPS is probably overestimated in these small studies, due to selection bias and non-structured surveillance. A recent prospective study in 41 patients showed that, under annual surveillance, none of the patients developed CRC during 5-year follow-up. ${ }^{19}$

Large multicentre studies are needed to estimate the actual CRC incidence under surveillance in daily practice. Moreover, it is important to characterise those patients with SPS at increased CRC risk, in order to enable personalised treatment and surveillance protocols. The aim of this study was to assess CRC risk factors in a large cohort of patients with SPS and to evaluate the overall risk of CRC during surveillance.

\section{METHODS}

\section{Study design and population}

This is a retrospective international multicentre cohort study. Patients were enrolled from seven centres in the Netherlands and two centres in the UK. In each participating centre, a search was performed for patients with multiple SPs in their medical history. Data were retrieved from medical charts, pathology and endoscopy reports and hereditary CRC databases to enable the registration of as much patients as possible. Data from 1993 to 2015 were included for analysis. Patients were included based on a retrospective polyp count of all lesions. Patients who fulfilled 2010 WHO criterion-1 and/or WHO criterion-3 for the diagnosis of SPS were eligible for inclusion in this study. Patients who only fulfilled WHO criterion-2 were excluded from the analysis. The clinical characteristics of these patients are presented in online supplementary appendix 2 separately. Patients with IBD and/or patients with a known germline adenomatous polyposis coli (APC) mutation, a known mutation in one of the mismatch repair genes (Lynch syndrome) or a known biallelic Mut-YH mutation were also excluded. The study protocol was presented to the local institutional review board (IRB) of the Academic Medical Centre for ethical approval. The IRB decided that formal revision was not required for this study, in agreement with the Dutch Medical Research Involving Human Subjects Act, since patient data were retrieved retrospectively and no additional interventions were performed for the purpose of this study. Formal ethical committee approval was not required for the UK sites for the same reasons as the Dutch cohort but each site sought and received local research and development department approval. This study was carried out in accordance with the Helsinki Declaration. ${ }^{20}$

\section{Clinical characteristics}

In each participating centre, data on patient age, gender, smoking status and body mass index (BMI) were gathered from medical charts. Medical charts were also used to gather patient and familial history records of CRC and extracolonic cancers. Colonoscopy reports and surgery reports with corresponding pathology reports were used to collect information regarding the number, size, location and type of colonic polyps detected per patient. These reports were also used to define the initial clinical presentation (symptomatic, familial risk or population screening), the number of colonoscopies, the time interval between colonoscopies, the date of SPS diagnosis and the type as well as the reason for surgical colonic resections, if applicable. Data were collected and stratified as follows: before SPS diagnosis, at SPS diagnosis or during surveillance. The surveillance period was defined as the period after complete endoscopic and/or surgical clearing of all clinically relevant SPs (all lesions above $5 \mathrm{~mm}$ ). As a result, lesions that were detected between the diagnosis of SPS and the clearing of all relevant polyps were included in the 'at diagnosis' group. All data were anonymised per centre before being uploaded to a central database.

\section{Histopathology}

Histopathology was considered as the reference standard in this study. No centralised histopathology revision was performed. All detected lesions were assigned as SPs, adenomas or as 'other type of polyp'. All HPs, SSA/Ps and TSAs were accounted as SPs but were not taken into account separately in the analyses, given the retrospective design of this study and the high interobserver variability between pathologists in the differentiation of these polyp subtypes. ${ }^{21} 22$ SPs were subdivided into SPs without dysplasia and those with dysplasia. Adenomas were subdivided into non-advanced adenomas and advanced adenomas. Adenomas $\geq 10 \mathrm{~mm}$, with high-grade dysplasia and/or a villous component were accounted as advanced adenomas.

\section{Study outcomes and statistical analysis}

The primary objective of this study was to evaluate clinical risk factors associated with CRC in patients with SPS. Both clinical risk factors as well as colonoscopy risk factors were evaluated in a per-patient analysis. In case of a median number of detected lesions per patient of $>0$, risk factors were handled as ordinal or continuous variable. In case of a median number of detected lesions of 0 , risk factors were dichotomised and treated as a binary variable. For each risk factor, the univariate association with the occurrence of CRC was calculated and presented as OR with 95\% CI. Multivariable logistic regression was used to assess the adjusted associations between these risk factors and the presence of CRC. All risk factors that showed a significant association with CRC in the univariate analysis were included in the multivariable analyses, as well as age at SPS diagnosis. Missing data were assumed to be missing at random. Multiple imputation, using a multivariable model, was performed to adjust for missing values. ${ }^{23}$ Analyses were performed using 10 
imputed datasets. A sensitivity analysis was performed, in which patients diagnosed with CRC before diagnosed with SPS were excluded. Secondary objective of this study was to evaluate the risk of developing CRC during surveillance after the clearing of all relevant lesions. Kaplan-Meier survival analysis was used to calculate the 5-year cumulative incidence for CRC. Furthermore, the incidence rate of CRC during surveillance was measured. Statistical analyses were performed using SPSS statistics (V.21; SPSS, Chicago, Illinois, USA) and R (V.2.15.0) (The R Foundation for Statistical Computing). A two-sided $\mathrm{p}$ value $<0.05$ was considered significant in the analyses.

\section{RESULTS}

\section{Patient characteristics}

In total, 480 patients were identified who fulfilled at least one of the WHO criteria for SPS (figure 1). Of these patients, only 27 fulfilled WHO criterion-2, 14 also had been diagnosed with IBD and 5 had a hereditary CRC syndrome (4 patients with Lynch syndrome and 1 with Mut-YH associated polyposis). These patients were excluded, resulting in a total of 434 patients eligible for inclusion in the analysis, of whom 283 patients (65.2\%) were diagnosed from 2010 onwards. Of these 434 patients, $292(67.3 \%)$ were included in a Dutch centre and 142 $(32.7 \%)$ in a British centre.

Baseline characteristics of patients are presented in table 1. The median age of patients at diagnosis was 60.8 years (IQR 51.7-67.0) and 211 patients (48.6\%) were male. In total, 117 patients (27.0\%) fulfilled WHO criterion-1 only, 179 patients (41.2\%) fulfilled WHO criterion-3 only and 138 patients (31.8\%) fulfilled both WHO criteria 1 and 3. First clinical presentation was: 'symptomatic' for 308 patients (71.0\%), 'familial cancer risk' for 69 patients (15.9\%) and 'population screening' for 57 patients (13.1\%). In total, 182 patients $(56.9 \%)$ had a history of smoking, 41 patients $(16.2 \%)$ had a BMI $\geq 30,149$ patients $(38.4 \%)$ had at least one FDR with CRC and 25 patients $(5.9 \%)$ had at least one FDR that was also diagnosed with SPS (WHO criterion-1 and/or WHO criterion-3).

The overall median number of detected SPs (detected up to diagnosis and during surveillance) per patient was 29 (IQR 1750). The median number of detected lesions was 14 (IQR 7-25) for SPs proximal to the rectosigmoid, 3 (IQR 1-5) for SPs $\geq 10 \mathrm{~mm}$ and $3(0-5)$ for SPs $\geq 10 \mathrm{~mm}$ proximal to the rectosigmoid. In total, 330 patients $(76.0 \%)$ were diagnosed with at

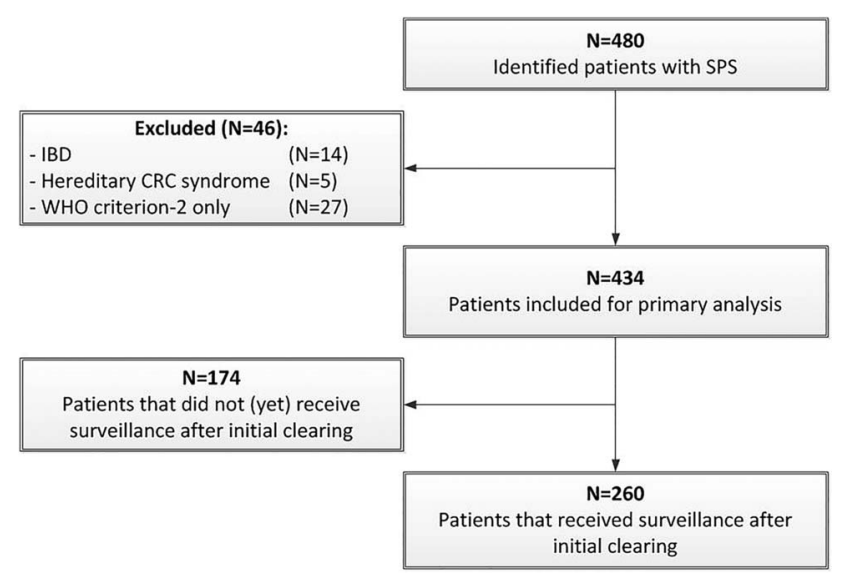

Figure 1 Study flowchart. CRC, colorectal cancer; SPS, serrated polyposis syndrome. least one SP $\geq 10 \mathrm{~mm}, 310$ patients $(71.4 \%)$ with at least one SP $\geq 10 \mathrm{~mm}$ proximal to the rectosigmoid and 114 patients (26.3\%) with at least one SP-containing dysplasia. The overall median number of detected adenomas per patient was 2 (IQR $0-5)$. In total, 324 patients $(74.7 \%)$ were also diagnosed with at least one adenoma and 153 patients (35.3\%) were diagnosed with at least one advanced adenoma.

Overall, 127/434 patients (29.3\%) were diagnosed with CRC, of whom $8 / 434$ patients $(1.8 \%)$ were diagnosed with two synchronous primary CRCs and 9/434 patients (2.1\%) with two metachronous CRCs (table 2). The median age at first diagnosis of CRC was 60.8 years (range 20.3-84.7). In total 33/117 patients (28.2\%) diagnosed with WHO criterion-1, 44/179 patients (24.6\%) diagnosed with WHO criterion-3 and 50/138 patients $(36.2 \%)$ diagnosed with both WHO criteria 1 and 3 were diagnosed with CRC $(p=0.07)$. With regard to first

Table 1 Baseline characteristics

\begin{tabular}{|c|c|}
\hline Total cohort size; $\mathrm{n}$ & 434 \\
\hline Age at diagnosis; median (IQR) & $60.8(51.7-67.0)$ \\
\hline Male gender; $\mathrm{n}(\%)$ & $211(48.6)$ \\
\hline \multicolumn{2}{|l|}{ WHO subtype; n (\%) } \\
\hline Type 1 & $117(27.0)$ \\
\hline Type 3 & $179(41.2)$ \\
\hline Type $1+3$ & $138(31.8)$ \\
\hline \multicolumn{2}{|l|}{ Smoking status; n (\%) } \\
\hline Current smoker & $126(39.4)$ \\
\hline Former smoker & $56(17.5)$ \\
\hline No smoker & $138(43.1)$ \\
\hline Missing & 114 \\
\hline \multicolumn{2}{|l|}{$\mathrm{BMl} ; \mathrm{n}(\%)$} \\
\hline$\geq 30 \mathrm{~kg} / \mathrm{m}^{2}$ & $41(16.2)$ \\
\hline$<30 \mathrm{~kg} / \mathrm{m}^{2}$ & $212(83.8)$ \\
\hline Missing & 181 \\
\hline \multicolumn{2}{|l|}{ FDR with CRC; $\mathrm{n}(\%)$} \\
\hline Yes & $149(38.4)$ \\
\hline No & $239(61.6)$ \\
\hline Missing & 46 \\
\hline \multicolumn{2}{|l|}{ FDR with SPS; $\mathrm{n}(\%)$} \\
\hline Yes & $25(5.9)$ \\
\hline No & $409(94.1)$ \\
\hline \multicolumn{2}{|l|}{ Clinical presentation; $\mathrm{n}(\%)$} \\
\hline Symptoms & $308(71.0)$ \\
\hline Familial cancer risk & $69(15.9)$ \\
\hline Population screening & $57(13.1)$ \\
\hline \multicolumn{2}{|l|}{ Overview of detected polyps per patient } \\
\hline No. of SPs; median (IQR) & $29(17-50)$ \\
\hline No. of SPs proximal to the rectosigmoid; median (IQR) & $14(7-25)$ \\
\hline No. of $S P s \geq 10 \mathrm{~mm}$; median (IQR) & $3(1-5)$ \\
\hline Patients with at least $1 \mathrm{SP} \geq 10 \mathrm{~mm} ; \mathrm{n}(\%)$ & $330(76.0)$ \\
\hline $\begin{array}{l}\text { No. of SPs } \geq 10 \mathrm{~mm} \text { proximal to the rectosigmoid; median } \\
\text { (IQR) }\end{array}$ & $3(0-5)$ \\
\hline $\begin{array}{l}\text { Patients with at least } 1 \mathrm{SP} \geq 10 \mathrm{~mm} \text { proximal to the } \\
\text { rectosigmoid; } \mathrm{n}(\%)\end{array}$ & $310(71.4)$ \\
\hline No. of SPs containing dysplasia; median (range) & $0(0-18)$ \\
\hline Patients with at least 1 SP-containing dysplasia; $n(\%)$ & $114(26.3)$ \\
\hline No. of adenomas; median (IQR) & $2(0-5)$ \\
\hline Patients with at least 1 adenoma; $\mathrm{n}(\%)$ & $324(74.7)$ \\
\hline No. of advanced adenomas; median (range) & $0(0-6)$ \\
\hline Patients with at least 1 advanced adenoma; $\mathrm{n}(\%)$ & $153(35.3)$ \\
\hline
\end{tabular}


Table 2 Characteristics of patients with CRC

\begin{tabular}{lc}
\hline All patients with CRC; $\mathrm{n}(\%)$ & $127(29.3)$ \\
Patients with two synchronous primary CRCs; $\mathrm{n}(\%)$ & $8(1.8)$ \\
Patients with metachronous CRC; $\mathrm{n}(\%)$ & $9(2.1)$ \\
Age at diagnosis first CRC; median (range) & $60.8(20.3-84.7)$ \\
WHO subtype; $\mathrm{n}(\%)$ & \\
$\quad$ Criterion-1 & $33(26.0)$ \\
Criterion-3 & $44(34.6)$ \\
Criterion 1+3 & $50(39.4)$ \\
Moment of diagnosis CRC (144 cancers); $\mathrm{n}(\%)^{*}$ & \\
Before diagnosis SPS & $74(51.4)$ \\
At diagnosis SPS & $68(47.2)$ \\
During surveillance & $2(1.4)$ \\
Location CRC (144 cancers); $\mathrm{n}(\%)$ & \\
Caecum & $14(9.7)$ \\
Ascending colon & $31(21.5)$ \\
Transverse colon & $24(16.7)$ \\
Descending colon & $6(4.2)$ \\
Rectosigmoid & $69(47.9)$ \\
\hline${ }^{*}$ Carcinomas detected between diagnosis of SPS and clearing of all relevant polyps \\
were included in the 'at diagnosis' group. \\
CRC, colorectal cancer; SPS, serrated polyposis syndrome.
\end{tabular}

clinical presentation, $13 / 57$ patients $(22.8 \%)$ who presented via population screening, $110 / 308$ patients $(35.7 \%)$ who presented with symptoms and $4 / 69$ patients $(5.8 \%)$ who were screened for a familial CRC risk were diagnosed with CRC $(\mathrm{p}<0.001)$. In total, 74 CRCs $(51.4 \%)$ were detected before the diagnosis of SPS, 68 CRCs $(47.2 \%)$ at the time of diagnosis of SPS and 2 CRCs (1.4\%) during SPS surveillance. In total, 75/144 CRCs $(52.1 \%)$ were located in the left-sided colon and 69/144 $(47.9 \%)$ in the right-sided colon. The different SPS phenotypes showed no statistically significant difference in the location of CRC $(p=0.53)$. In total 69/144 CRCs $(47.9 \%)$ were diagnosed in the rectosigmoid. Median age at diagnosis of CRC in these patients was 58.0 (range 20.3-79.2) versus 63.7 (range 26.984.7) for patients diagnosed with CRC proximal to the rectosigmoid $(p=0.03)$. No difference was found for gender $(48.5 \%$ vs $37.3 \%$ male; $\mathrm{p}=0.20)$.

\section{Risk factors for CRC}

Risk factors for CRC in patients with SPS are presented in table 3. Univariate analyses showed an association with CRC in patients who were diagnosed with at least one SP-containing dysplasia (OR 2.34; 95\% CI 1.48 to $3.70 ; \mathrm{p}<0.001$ ) and for patients with at least one advanced adenoma (OR 2.46; 95\% CI 1.59 to $3.80 ; \mathrm{p}<0.001)$. Patients who fulfilled both WHO criteria 1 and 3 also showed an increased CRC risk (OR 1.62; $95 \%$ CI 1.05 to $2.49 ; \mathrm{p}=0.03$ ). The cumulative number of SPs as well as adenomas was not significantly associated with the diagnosis of CRC. Patients with CRC were significantly older at SPS diagnosis when compared with patients without CRC $(\mathrm{p}<0.001)$. A history of smoking was inversely associated with CRC (OR 0.48; 95\% CI 0.30 to 0.78 ; $\mathrm{p}<0.01$ ).

Multiple logistic regression analysis showed similar results. Patients having at least one SP with dysplasia (OR 2.07; 95\% CI 1.28 to $3.33 ; \mathrm{p}<0.01$ ), at least one advanced adenoma (OR $2.30 ; 95 \%$ CI 1.47 to $3.67 ; \mathrm{p}<0.001)$ and patients who fulfilled both WHO criteria 1 and 3 (OR 1.60; 95\% CI 1.04 to 2.51; $\mathrm{p}<0.05$ ) had an increased risk to be diagnosed with CRC, adjusted for the confounder of age at SPS diagnosis. Patients with a history of smoking had a lower risk of CRC (OR 0.36; $95 \%$ CI 0.23 to $0.56 ; p<0.001)$. The decreased risk of CRC in patients with a history of smoking was found both for women (adjusted OR 0.49 ; 95\% CI 0.26 to 0.95 ) and for men (adjusted OR $0.30 ; 95 \%$ CI 0.15 to 0.60$)$. Subsequent analysis showed that these patients significantly more often fulfilled WHO criterion-3 only (OR 1.73 ; 95\% CI 1.10 to $2.74 ; \mathrm{p}=0.02$ ). The performed sensitivity analysis showed no structural differences in the association between potential risk factors and CRC.

\section{Clinical management of SPS}

Of the 434 patients, 403 (92.9\%) received clearing of all clinically relevant lesions, 28 patients were not yet cleared and 3 patients died from CRC before clearing was accomplished (table 4). The median time from diagnosis up to clearing of all relevant lesions was 1.5 months (IQR 0-8.7). In total, 95/403 patients $(23.6 \%)$ needed surgery during the clearing phase, of which $56(58.9 \%)$ was due to CRC, $30(31.6 \%)$ was due to polyp burden, $8(8.4 \%)$ was due to an unresectable polyp and 1

Table 3 Risk factors for CRC in 434 patients with SPS

\begin{tabular}{|c|c|c|c|c|c|c|}
\hline & $\begin{array}{l}\text { Patients with } \\
\text { CRC }(n=127)\end{array}$ & $\begin{array}{l}\text { Patients without } \\
\text { CRC }(n=307)\end{array}$ & $\begin{array}{l}\text { Univariate } \\
\text { OR }(95 \% \mathrm{CI})\end{array}$ & p Value & $\begin{array}{l}\text { Multivariable } \\
\text { OR }(95 \% \mathrm{CI})\end{array}$ & p Value \\
\hline Age at diagnosis SPS in years; median (IQR) & $63.6(58.1-69.6)$ & $59.4(48.2-65.4)$ & 1.04 (1.02 to 1.06$)$ & $<0.001$ & 1.03 (1.01 to 1.05$)$ & $<0.001$ \\
\hline Male gender; $\mathrm{n}(\%)$ & $55(43.3)$ & $156(50.8)$ & $0.74(0.49$ to 1.12$)$ & 0.16 & & \\
\hline A history of smoking; $\mathrm{n}(\%)^{*}$ & $43(44.3)$ & $139(62.3)$ & $0.48(0.30$ to 0.78$)$ & $<0.01$ & $0.36(0.23$ to 0.56$)$ & $<0.001$ \\
\hline $\mathrm{BMI} \geq 30 ; \mathrm{n}(\%)^{*}$ & $9(10.6)$ & $32(19.0)$ & $0.50(0.22$ to 1.07$)$ & 0.09 & & \\
\hline At least one FDR with CRC; $n(\%)^{*}$ & $38(34.2)$ & $111(40.1)$ & 0.78 (0.49 to 1.23$)$ & 0.29 & & \\
\hline At least one FDR with SPS; $n$ (\%) & $5(4.1)$ & $20(6.7)$ & $0.60(0.20$ to 1.51$)$ & 0.31 & & \\
\hline No. of SPs; median (IQR) & $29(18-46)$ & $29(17-52)$ & 1.00 (0.99 to 1.01$)$ & 0.67 & & \\
\hline No. of SPs $\geq 10 \mathrm{~mm}$; median (IQR) & $3(1-5)$ & $3(1-5)$ & 0.99 (0.96 to 1.03$)$ & 0.69 & & \\
\hline No. of SPs proximal to the rectosigmoid; median (IQR) $\dagger$ & $15(8-27)$ & $13(7-25)$ & 1.00 (0.99 to 1.01$)$ & 0.94 & & \\
\hline No. of $S P s \geq 10 \mathrm{~mm}$ proximal to the rectosigmoid; median (IQR) $\dagger$ & $3(1-5)$ & $2(1-4)$ & $1.00(0.96$ to 1.04$)$ & 0.99 & & \\
\hline At least 1 SP containing dysplasia; $n(\%)$ & $48(37.8)$ & $66(21.5)$ & $2.34(1.48$ to 3.70$)$ & $<0.001$ & 2.07 (1.28 to 3.33$)$ & $<0.01$ \\
\hline No. of adenomas; median (IQR) & $2(1-5)$ & $2(0-5)$ & $1.03(0.98$ to 1.08$)$ & 0.30 & & \\
\hline At least 1 advanced adenoma; n (\%) & $64(50.4)$ & $89(29.0)$ & 2.46 (1.59 to 3.80$)$ & $<0.001$ & 2.30 (1.47 to 3.67$)$ & $<0.001$ \\
\hline Fulfilling WHO criteria 1 and 3; n (\%) & $50(39.4)$ & $88(28.7)$ & $1.62(1.05$ to 2.49$)$ & 0.02 & $1.60(1.04$ to 2.51$)$ & $<0.05$ \\
\hline
\end{tabular}


Table 4 Clinical management of patients with serrated polyposis syndrome

\begin{tabular}{|c|c|}
\hline No. of patients who received clearing of all relevant lesions & 403 \\
\hline Time from diagnosis up to clearing (months); median (IQR) & $1.5(0-8.7)$ \\
\hline No. of patients who received surgery during clearing; $n(\%)$ & $95(23.6)$ \\
\hline \multicolumn{2}{|l|}{ Reason surgery during clearing; $\mathrm{n}(\%)$} \\
\hline CRC & $56(58.9)$ \\
\hline Polyp burden & $30(31.6)$ \\
\hline Unresectable polyp & $8(8.4)$ \\
\hline Perforation due to polypectomy & $1(1.1)$ \\
\hline No. of needed clearing colonoscopies; median (IQR)* & $2(1-3)$ \\
\hline $\begin{array}{l}\text { No. of patients who received surveillance after clearing of all } \\
\text { polyps }\end{array}$ & 260 \\
\hline $\begin{array}{l}\text { Follow-up time since clearing of all polyps (years); median } \\
\text { (IQR) } \dagger\end{array}$ & $3.2(1.6-5.7)$ \\
\hline No. of colonoscopies during surveillance; median (IQR) $\dagger$ & $2(1-4)$ \\
\hline $\begin{array}{l}\text { Interval between surveillance colonoscopies (years); median } \\
\text { (IQR) } \dagger\end{array}$ & $1.2(1.0-1.6)$ \\
\hline No. of patients who received surgery during surveillance; $n(\%)$ & $11(4.2)$ \\
\hline \multicolumn{2}{|l|}{ Reason surgery during surveillance; $\mathrm{n}(\%)$} \\
\hline CRC & $1(9.1)$ \\
\hline Polyp burden & $5(45.4)$ \\
\hline Unresectable polyp & $4(36.4)$ \\
\hline Perforation due to polypectomy & $1(9.1)$ \\
\hline \multicolumn{2}{|l|}{ Overview of detected polyps during surveillance per patient ${ }^{\dagger}$} \\
\hline No. of SPs; median (IQR) & $7(2-18)$ \\
\hline No. of SPs proximal to the rectosigmoid; median (IQR) & $2(0-7)$ \\
\hline No. of large SPs; median (IQR) & $0(0-1)$ \\
\hline Patients with at least $1 \mathrm{SP} \geq 10 \mathrm{~mm} ; \mathrm{n}(\%)$ & $70(26.9)$ \\
\hline No. of adenomas; median (IQR) & $0(0-2)$ \\
\hline Patients with at least 1 adenoma; $\mathrm{n}(\%)$ & $122(46.9)$ \\
\hline $\begin{array}{l}\text { Patients with at least } 1 \mathrm{SP} \geq 10 \mathrm{~mm} \text { proximal to the } \\
\text { rectosigmoid; } \mathrm{n}(\%)\end{array}$ & $65(25.0)$ \\
\hline Patients with at least 1 SP-containing dysplasia; n (\%) & $16(6.2)$ \\
\hline Patients with at least 1 advanced adenoma; n (\%) & $20(7.7)$ \\
\hline
\end{tabular}

* Referred to the 308 patients who did not receive surgery during clearing. tReferred to the 260 patients who received surveillance after clearing of all polyps. CRC, colorectal cancer; SPs, serrated polyps.

(1.1\%) was as a result of a perforation caused by polypectomy. For those 308 patients who could be treated endoscopically, a median number of 2 clearing colonoscopies (IQR 1-3) were needed.

A total of 260 patients (59.9\%) underwent surveillance after clearing of all lesions $>5 \mathrm{~mm}$ with a median follow-up of 3.2 years (IQR 1.6-5.7) and a median interval between colonoscopies of 1.2 years (IQR 1.0-1.6) (table 4). As mentioned, two patients were diagnosed with CRC during surveillance, which corresponds to an incidence rate of 1.9 events/1000 personyears of surveillance (95\% CI 0.3 to 6.4). The 5 -year cumulative incidence for CRC during surveillance was 1.5\% (95\% CI 0\% to $3.7 \%)$. The clinical characteristics of the two patients who developed CRC during surveillance are presented in online supplementary appendix 1 . A total of $11 / 260$ patients $(4.2 \%)$ needed surgery during surveillance: 1 patient due to CRC, 5 patients due to polyp burden, 4 patients due to an unresectable polyp and 1 patient as result of a perforation after polypectomy.

\section{DISCUSSION}

In this large multicentre study, we evaluated risk factors associated with CRC in patients with SPS and assessed the overall risk of developing CRC during surveillance. The presence of at least one SP-containing dysplasia, at least one advanced adenoma and/or a combined WHO criteria 1 and 3 phenotype was associated with CRC. Conversely, patients with a history of smoking showed an inverse association with CRC, possibly due to a different pathogenesis of disease. The incidence rate of CRC during surveillance and after clearing of all relevant lesions was 1.9 events/1000 person-years of surveillance $(95 \%$ CI 0.3 to 6.4 ), corresponding to a 5 -year cumulative risk of $1.5 \%$ (95\% CI $0 \%$ to $3.7 \%$ ).

In the current study, analyses were performed in the largest cohort of patients to date, enabling robust estimates for both CRC risk factors as well as CRC incidence during surveillance. This study has an international multicentre cohort design and included both patients from academic and from non-academic hospitals in the Netherlands and the UK. Nevertheless, several limitations have to be acknowledged. First, this study has a retrospective design. As a result, quality assurance of retrieved data was limited and this study might be subject to certain ascertainment bias in patient selection. Also, due to the retrospective design of the study, an established, uniform surveillance protocol was not in place, which may have introduced another element of bias. Despite the challenge of missing data, multiple imputation enabled the evaluation of all patients in the multivariable analysis. Second, due to logistic heterogeneity within centres, we were unable to perform a uniform search within the participating centres. Most centres already had a welldocumented prospective registry of SPS patients. These patients form the vast majority in the analysis. In the other centres, local computer systems were used to identify patients with multiple SPs. For these centres, certain patients might have been missed, imposing a risk of selection bias. A third limitation in this study is the fact that histopathology of the colonic lesions was not revised centrally by a panel of gastrointestinal pathologists. Several studies showed that the interobserver agreement between pathologists in the differentiation of SSA/Ps and HPs is considered moderate to low. ${ }^{21} 222425$ Therefore, individual analysis for the risk of SSA/Ps and HPs separately would probably have resulted in biased and unreliable results. To overcome this limitation, we have decided to appraise all SSA/Ps, HPs as well as TSAs as identical lesions and to perform an overarching analysis based on polyp location, size and the presence of dysplasia. The same decision was made in the most recent European Society of Gastrointestinal Endoscopy guideline for post-polypectomy surveillance after the resection of SPs. ${ }^{26}$ The presence of dysplasia as well as polyp size was taken into account in this guideline. However, due to the same histopathological restrictions, surveillance intervals were not based on histopathological subclassification. Taken these considerations into account, the external validity of this study seems to be reasonably founded.

Several studies have reported on the risk of CRC in patients with SPS, demonstrating a variable risk, ranging from $7 \%$ to $70 \% .^{14} 1718{ }^{27-31}$ The overall prevalence of CRC in our study (29\%) was largely comparable with the risk that was presented in the three largest cohorts till date $(26 \%-29 \%) .{ }^{14} \quad 27 \quad 30$ However, due to the retrospective design of these studies and imposed selection bias, the CRC prevalence in these studies probably is a poor proxy for the real prevalence of disease. In a subsequent analysis, we demonstrated that the risk of CRC at presentation largely depends on the first clinical presentation. In total, $22.8 \%$ of patients who presented via population screening, $35.7 \%$ of patients who presented with symptoms and $5.8 \%$ of patients who were identified due to a familial CRC risk were diagnosed with CRC $(\mathrm{p}<0.001)$. These results suggest that the 
overall CRC risk in not-yet-diagnosed asymptomatic patients with SPS is probably overestimated in previous studies as well as in the current report. ${ }^{14} 17{ }^{18}{ }^{27-31}$ Few studies have tried to risk stratify SPS patients based on clinical risk factors. ${ }^{30}{ }^{31} \mathrm{In}$ a large cohort of 115 patients with SPS, the risk of CRC was evaluated, stratified for phenotype: few large right-sided polyps, many small left-sided polyps or a pancolonic phenotype. ${ }^{30}$ These phenotypes largely overlap with those presented in the current study. In this study a significant difference for CRC risk between the described phenotypes could not be detected. ${ }^{30}$ However, we demonstrated that patients who fulfilled both WHO criteria 1 and 3 were at increased risk to be diagnosed with CRC, compared with patients who fulfilled WHO criterion-1 or WHO criterion-3 only (adjusted OR 1.60 ; 95\% CI 1.04 to 2.51; $\mathrm{p}<0.05$ ). In a large cross-sectional study, risk factors of CRC were evaluated in 151 patients with at least five SPs outside the rectum and most of these patients probably fulfilled the WHO criteria for SPS. ${ }^{32}$ CRC was diagnosed in 57 patients (38\%). Current smokers showed a markedly decreased risk of CRC compared with non-smokers (OR 0.35; 95\% CI 0.15 to 0.82 ). The authors described this phenomenon as 'the smoking paradox'. One of the potential explanations for this paradox is the hypothesis that the effect of smoking may be mainly observed on the development of diminutive polyps. ${ }^{32}$ Therefore, the risk in a given population of patients with increased CRC risk may be smaller in the smokers than in the non-smokers. This hypothesis is supported by the fact that in the current study patients with a history of smoking (current and former smokers) significantly more often fulfilled WHO criterion-3 only, compared with non-smokers (OR 1.7; 95\% CI 1.1 to $2.7 ; \mathrm{p}=0.02$ ). This suggests that the pathogenesis of SPS might be different in smokers and non-smokers, which might influence the options for therapy and surveillance for smokers in the near future. Unfortunately, due to the retrospective design of this study, a reliable differentiation between current and former smokers could not be made.

Finally, we showed that the incidence of CRC during nonstructured surveillance is probably lower than assumed in one of our earlier reports. ${ }^{14}$ The 5 -year cumulative incidence of CRC during surveillance was $1.5 \%$ versus $6.5 \%$ that we have reported earlier. ${ }^{14}$ Contrary to our prior study, CRCs diagnosed within the period between SPS diagnosis and the clearing of all relevant lesions were not accounted as cancers developed during surveillance. ${ }^{14}$ This strategy aligns with international protocols, which advice to clear all relevant lesions before the start of CRC surveillance. ${ }^{11} 12$ This study confirms earlier results from our group, showing that annual colonoscopy surveillance after the resection of all clinically relevant lesions is relatively safe. ${ }^{19}$

The results from this study raise questions about some aspects of the current WHO guidelines for the diagnosis of SPS. ${ }^{10}$ In the current WHO guideline, rectal lesions are excluded from the clinical diagnostic criteria and in criteria 1 and 2 lesions in the sigmoid colon are excluded. This seems mainly driven by the fact that diminutive HPs in the rectosigmoid should probably not be taken into account for the diagnosis of SPS. However, results from our study showed that $47.9 \%$ of all CRCs were located in the rectosigmoid. These cancers were detected in patients who were significantly younger than patients with CRCs located proximal to the rectosigmoid (median 58.0 vs $63.7 ; \mathrm{p}=0.03$ ). It is not possible to exclude that a proportion of these CRCs may have arisen from an adenoma rather than a SP but the substantial proportion of the CRC arising in the rectosigmoid at a young age implies that those SPs located in the rectosigmoid are of clinical importance. It would seem reasonable then to re-assess the WHO criteria and not exclude lesions purely on their location without taking into account their size and histopathology. Hopefully these adjustments to the current WHO guidelines could help in assigning those patients who are truly at risk of developing CRC.

Future studies should mainly focus on the safety and feasibility of personalised treatment and surveillance for patients with SPS in order to decrease patient burden as well as the incidence of colonoscopy interval CRCs. The clinical risk factors, as described in this study, could potentially help to risk stratify patients with SPS for different surveillance intervals. However, the low risk of CRC during surveillance could also argue for prolonged surveillance intervals, for those individuals with and without CRC risk factors. Furthermore, research should be conducted to evaluate the effect of the discontinuation of smoking on the polyp burden in patients with SPS. A potential beneficial result could contribute to provide assistance for patients to quit smoking.

In conclusion, we showed that SPs containing dysplasia, advanced adenomas and/or a combined WHO criteria 1 and 3 phenotype are associated with CRC in patients with SPS, while a history of smoking is inversely associated with CRC in these patients. Furthermore, we showed that the risk of developing CRC during non-structural surveillance is lower than earlier assessed in literature. Future research should focus on the safety and feasibility of personalised treatment and surveillance for patients with SPS, in order to decrease patient burden as well as the number of colonoscopy interval CRCs.

\section{Author affiliations}

${ }^{1}$ Department of Gastroenterology and Hepatology, Academic Medical Centre, University of Amsterdam, Amsterdam, The Netherlands

${ }^{2}$ The Polyposis Registry, St Mark's Hospital, London, UK

${ }^{3}$ Translational Gastroenterology Unit, Experimental Medicine Division, Nuffield Department of Clinical Medicine, University of Oxford, John Radcliffe Hospital, Oxford, UK

${ }^{4}$ Department of Gastroenterology and Hepatology, Radboud University Medical Centre, Nijmegen, The Netherlands

${ }^{5}$ Department of Gastroenterology and Hepatology, Netherlands Cancer Institute, Amsterdam, The Netherlands

${ }^{6}$ Division of Gastroenterology and Hepatology, GROW School for Oncology and Developmental Biology, Maastricht University Medical Centre, Maastricht, The Netherlands

${ }^{7}$ Department of Gastroenterology and Hepatology, Erasmus University Medical Centre, Rotterdam, The Netherlands

${ }^{8}$ Department of Pathology, Netherlands Cancer Institute, Amsterdam, The Netherlands

${ }^{9}$ Department of Gastroenterology and Hepatology, St. Antonius Hospital, Nieuwegein, The Netherlands

${ }^{10}$ Department of Gastroenterology and Hepatology, University Medical Centre Groningen, University of Groningen, Groningen, The Netherlands

${ }^{11}$ Department of Pathology, Radboud University Medical Centre, Nijmegen, The Netherlands

Contributors All authors have contributed to this article in the following way: substantial contributions to the conception or design of the work, or the acquisition, analysis or interpretation of data. Drafting the work or revising it critically for important intellectual content. Final approval of the version published. Agreement to be accountable for all aspects of the work in ensuring that questions related to the accuracy or integrity of any part of the work are appropriately investigated and resolved. Conception and design: JEGI, ED; data acquisition: all authors; data analysis and interpretation: all authors; drafting the manuscript: JEGl; critical revision of the manuscript: all authors; statistical analysis: JEGI; supervision: all authors.

Funding KWF Kankerbestrijding (grant no. UVA 2014-7500).

Competing interests None declared.

Provenance and peer review Not commissioned; externally peer reviewed. 


\section{REFERENCES}

1 Siegel R, Naishadham D, Jemal A. Cancer statistics, 2012. CA Cancer J Clin 2012;62:10-29.

2 Zauber AGAAG, Winawer SSJ, O'Brien MJ, et al. Colonoscopic polypectomy and long-term prevention of colorectal-cancer deaths. N Engl J Med 2012;67:355-6.

3 Samowitz WS, Albertsen H, Herrick J, et al. Evaluation of a large, population-based sample supports a $\mathrm{CpG}$ island methylator phenotype in colon cancer. Gastroenterology 2005;129:837-45.

4 Toyota M, Ahuja N, Ohe-Toyota M, et al. CpG island methylator phenotype in colorectal cancer. Proc Natl Acad Sci USA 1999;96:8681-6.

5 Jass JR. Classification of colorectal cancer based on correlation of clinical, morphological and molecular features. Histopathology 2007;50:113-30.

6 East JE, Saunders BP, Jass JR. Sporadic and syndromic hyperplastic polyps and serrated adenomas of the colon: classification, molecular genetics, natural history, and clinical management. Gastroenterol Clin North Am 2008;37:25-46.

7 Leggett $B$, Whitehall $V$. Role of the serrated pathway in colorectal cancer pathogenesis. Gastroenterology 2010;138:2088-100.

8 Snover DC. Update on the serrated pathway to colorectal carcinoma. Hum Pathol 2011:42:1-10

9 Boparai KS, Dekker E, Polak MM, et al. A serrated colorectal cancer pathway predominates over the classic WNT pathway in patients with hyperplastic polyposis syndrome. Am J Pathol 2011;178:2700-7.

10 Snover DC, Ahnen DJ, Burt RW, et al. Serrated polyps of the colon and rectum and serrated polyposis. In: Bosman T, Carneiro F, Hruban R, et al. eds. WHO classification of tumours of the digestive system. Lyon, 2010. 160-5.

11 Rex DK, Ahnen DJ, Baron JA, et al. Serrated lesions of the colorectum: review and recommendations from an expert panel. Am J Gastroenterol 2012;107:1315-29; quiz 1314, 1330.

12 Rosty C, Hewett DG, Brown IS, et al. Serrated polyps of the large intestine: current understanding of diagnosis, pathogenesis, and clinical management. J Gastroenterol 2013;48:287-302.

13 East JE, Vieth M, Rex DK. Serrated lesions in colorectal cancer screening: detection, resection, pathology and surveillance. Gut 2015;64:991-1000.

14 Boparai KS, Mathus-Vliegen EMH, Koornstra JJ, et al. Increased colorectal cancer risk during follow-up in patients with hyperplastic polyposis syndrome: a multicentre cohort study. Gut 2010;59:1094-100.

15 Edelstein DL, Axilbund JE, Hylind LM, et al. Serrated polyposis: rapid and relentless development of colorectal neoplasia. Gut 2013;62:404-8.

16 Ferrández A, Samowitz W, DiSario JA, et al. Phenotypic characteristics and risk of cancer development in hyperplastic polyposis: Case series and literature review. Am J Gastroenterol 2004;99:2012-18.

17 Hyman NH, Anderson P, Blasyk H. Hyperplastic polyposis and the risk of colorectal cancer. Dis Colon Rectum 2004;47:2101-4.
18 Rubio CA, Stemme S, Jaramillo E, et al. Hyperplastic polyposis coli syndrome and colorectal carcinoma. Endoscopy 2006;38:266-70.

19 Hazewinkel Y, Tytgat KM, van Eeden S, et al. Incidence of colonic neoplasia in patients with serrated polyposis syndrome who undergo annual endoscopic surveillance. Gastroenterology 2014;147:88-95.

20 World Medical Association. World Medical Association Declaration of Helsinki: ethical principles for medical research involving human subjects. JAMA 1997;310:2191-4.

21 Ensari A, Bilezikçi B, Carneiro F, et al. Serrated polyps of the colon: how reproducible is their classification? Virchows Arch 2012:461:495-504.

22 Rau TT, Agaimy A, Gehoff A, et al. Defined morphological criteria allow reliable diagnosis of colorectal serrated polyps and predict polyp genetics. Virchows Arch 2014;464:663-72.

23 Streiner DL. The case of the missing data: methods of dealing with dropouts and other research vagaries. Can J Psychiatry 2002;47:68-75.

24 Abdeljawad K, Vemulapalli KC, Kahi CJ, et al. Sessile serrated polyp prevalence determined by a colonoscopist with a high lesion detection rate and an experienced pathologist. Gastrointest Endosc 2015;81:517-24.

25 Aust DE, Baretton GB. Serrated polyps of the colon and rectum (hyperplastic polyps, sessile serrated adenomas, traditional serrated adenomas, and mixed polyps)-proposal for diagnostic criteria. Virchows Arch 2010;457:291-7

26 Hassan C, Quintero E, Dumonceau J-M, et al. Post-polypectomy colonoscopy surveillance: European Society of Gastrointestinal Endoscopy (ESGE) Guideline. Endoscopy 2013;45:842-51

27 Chow $E$, Lipton $L$, Lynch $E$, et al. Hyperplastic polyposis syndrome: phenotypic presentations and the role of MBD4 and MYH. Gastroenterology 2006;131:30-9.

28 Lage $P$, Cravo M, Sousa $R$, et al. Management of Portuguese patients with hyperplastic polyposis and screening of at-risk first-degree relatives: a contribution for future guidelines based on a clinical study. Am J Gastroenterol 2004:99:1779-84.

29 Guarinos C, Sánchez-Fortún C, Rodríguez-Soler M, et al. Clinical subtypes and molecular characteristics of serrated polyposis syndrome. Clin Gastroenterol Hepatol 2013;11:705-11; quiz e46.

30 Kalady MF, Jarrar A, Leach $B$, et al. Defining phenotypes and cancer risk in hyperplastic polyposis syndrome. Dis Colon Rectum 2011;54:164-70.

31 Rosty C, Buchanan DD, Walsh MD, et al. Phenotype and Polyp Landscape in Serrated Polyposis Syndrome: a series of 100 patients from genetics clinics. Am J Surg Pathol 2012;36:876-82.

32 Buchanan DD, Sweet K, Drini M, et al. Risk factors for colorectal cancer in patients with multiple serrated polyps: a cross-sectional case series from genetics clinics. PLOS ONE 2010:5:1-7. 